\title{
ARTÍGULO ORIGINAL: Caracterización epide- miológica de los casos confirmados de influenza $A$ H1N1 a en el Hospital San Juan de Dios entre los meses de agosto 2009 a julio 2010
}

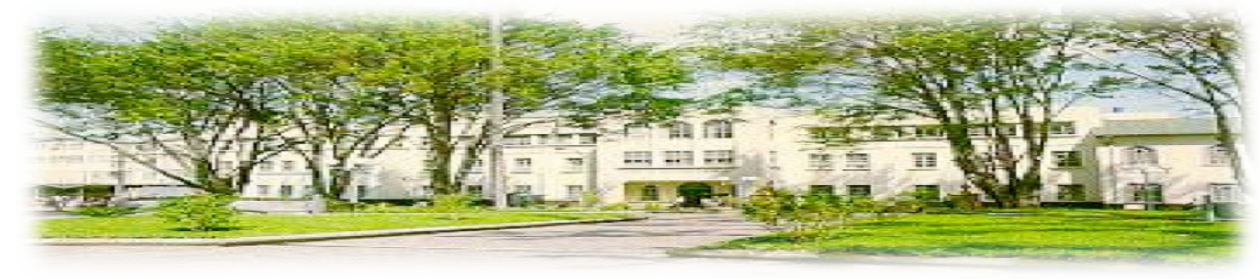

Hospital San quan de Dias. San José, Casta Rica. Fundado en 1845

\section{Comunicación Breve}

Recibido: $\quad 15 / 03 / 2014$

Aceptado: $\quad 26 / 11 / 2014$

Grupo de Trabajo Influenza AH1N1. Hospital San Juan de Dios. C.C.S.S

\section{INTRODUCCIÓN}

En el mes de abril de 2009 se hizo el anuncio de la presencia en México ${ }^{(1)}$ de casos de Influenza AH1N1 cuyos efectos eran poco conocidos en el ser humano. Dos semanas después de haberse detectado el brote, se había contabilizado 260 casos y 12 defunciones, lo que llevó a la cancelación de actividades públicas y vuelos en este país ${ }^{(2)}$.

Una de las situaciones más importantes con relación a esta epidemia es que no se conocía en sus orígenes las consecuencias ni la forma en que el virus atacaría a la población mundial y se desconocía si sería altamente patógeno, como el virus de Influenza AH5N1. Por este motivo las autoridades políticas y de salud a nivel mundial dieron la voz de alerta en cada uno de sus países para identificar posibles casos de esta enfermedad, la cual podría potencialmente causar una situación de emergencia sin precedentes en la población.

En Costa Rica, el primer caso probable se identificó el 28 de abril de $2009^{(3)}$. De acuerdo con el Boletín Semanal de Vigilancia de la Salud emitido por el Ministerio de Salud, a la semana $31^{(4)}$ se había notificado un total de 3738 casos confirmados y 71 defunciones asociadas al virus AH1N1.

Todas las redes de atención de servicios de salud del país afrontaron el embate de este novedoso virus, con los temores relacionados a la falta de información sobre su comportamiento y consecuencias. El Hospital San Juan de Dios (HSJD), fue uno de los centros con la responsabilidad de recibir casos sospechosos de Influenza por AH1N1. Este Hospital es uno de los centros de referencia especializados en Costa Rica, por lo que describir las características de los pacientes internados ayudaría a conocer el comportamiento clínico del virus.

El objetivo de este trabajo es caracterizar en tiempo, lugar y persona los casos confirmados de influenza AH1N1 y sus factores de riesgo en los pacientes internados en el HSJD entre los meses de agosto 2009 a julio 2010. 


\section{MÉTODOS}

Estudio descriptivo de los pacientes internados en el HSJD con el diagnóstico de infección por Influenza AH1N1, confirmados mediante reacción en cadena de polimerasa en tiempo real (PCR-rt) desde el 1 de agosto de 2009 hasta el 30 de julio del 2010.

La información se obtuvo del registro de pacientes hospitalizados por Influenza AH1N1 y del registro de vigilancia epidemiológica (SISVE) de la Caja Costarricense del Seguro Social.

Las variables de estudio fueron: edad, categorizada en tres grupos: de 20 a 39 años, de 40 a 59 años y de 60 y más años, sexo, tiempo transcurrido entre la fecha de inicio de síntomas y la primera consulta en cualquier centro de salud, tiempo transcurrido entre la fecha de inicio de síntomas y la fecha de internamiento en el HSJD, tiempo transcurrido entre la primera consulta y la fecha de internamiento en el HSJD, factor de riesgo, categorizados en 1) asma, 2) obesidad, 3) cardiopatía, 4) enfermedad pulmonar obstructiva crónica (EPOC), 5) embarazo, 6) diabetes mellitus tipo 2, 7) tabaquismo y 8) otros.
Se calculó las distribuciones de frecuencias, medidas de tendencia central y dispersión, tasa de ataque general (número de casos entre egresos al final del periodo por cien), razones $\mathrm{y}$ proporciones para la presentación de los resultados y su análisis.

Para el análisis se utilizó el software Excel 8.0, Epi-Info 3.5.1. e InfoSTAT.

\section{RESULTADOS}

Durante el periodo de estudio fueron internados 258 pacientes con diagnóstico de sospecha de influenza AH1N1. De éstos, 53 fueron los pacientes confirmados y la tasa de ataque general fue de $0.16 \%$.

La distribución de los pacientes confirmados de acuerdo a la semana epidemiológica en que fueron internados se puede observar en el gráfico 1 . Se denota en el mismo un patrón de fuente propagada. La mayoría de los casos se concentran de la semana 32 del año 2009 a la semana 7 del 2010 .

\section{Gráfico 1. Curva epidemiológica de casos confirmados con influenza AH1N1 según semana epidemiológica Hospital San Juan de Dios, agosto 2009 a julio 2010}

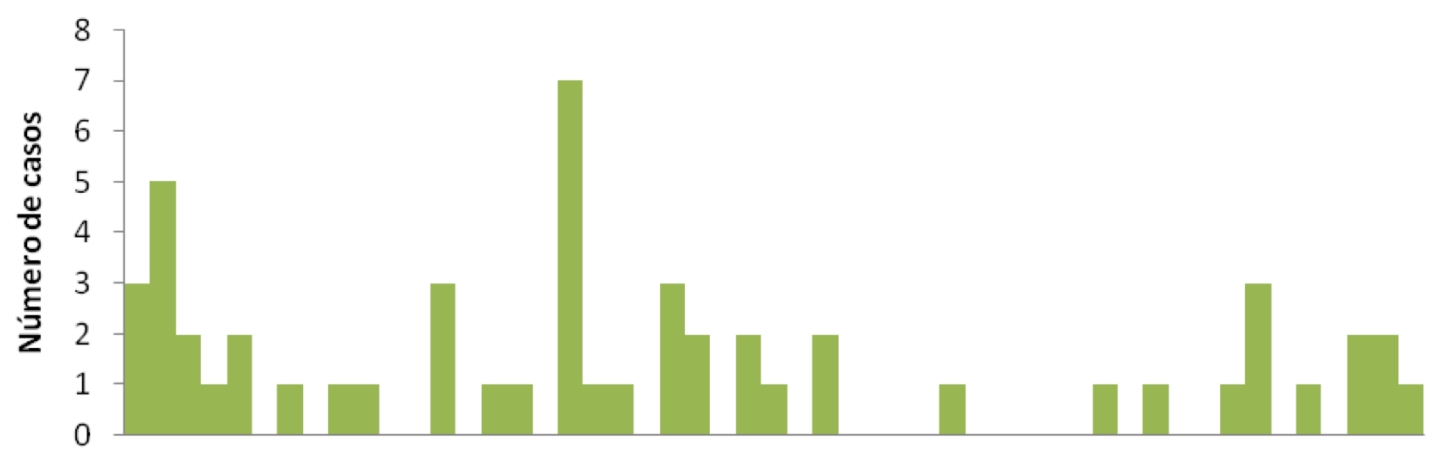

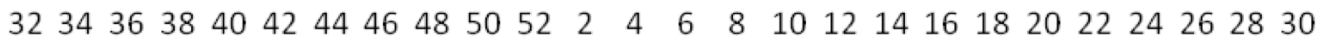

Semana epidemiológica

La mediana del tiempo que transcurrió entre el inicio de síntomas y la primera consulta fue de 2 días, con un intervalo intercuartil de 2 a 4 días, un rango de 0 a 31 días y una moda de 2 días.
En el caso del tiempo entre el inicio de síntomas y el ingreso al HSJD se obtuvo una mediana de 3 días, un intervalo intercuartil de 2 a 7 días, un rango de 0 a 31 días. La mitad de los pacientes 
que se internaron en el HSJD consultaron por primera vez el mismo día en que fueron internados. El rango de este periodo fue de 0 a 16 días.

En cuanto a la distribución según grupos de edad por sexo (Tabla 1) se observó una razón de masculinidad de uno a uno (1:1). La mediana de la edad de los pacientes fue de 52 años, con un rango de 21 a 88 años.

En cuanto a la distribución de los factores de riesgo por grupos de edad (gráfico 2), se observó que los más frecuentes en toda la población de estudio fueron diabetes $(n=19)$, que correspondió a un $35.8 \%$, Asma $(n=16)$ con un $30.2 \%$ y Obesidad $(n=12)$ igual a un $22.6 \%$.

Los pacientes con edades de 20 a 39 y 40 a 59 años no tuvieron EPOC como un factor de riesgo. El grupo de adultos jóvenes (20 a 39 años) no presentó pacientes con diagnóstico de cardiopatía o diabetes mellitus tipo 2. En total 10 pacientes (18.9\%) no presentaron ningún factor de riesgo. Durante el periodo en estudio se registró únicamente 2 pacientes embarazadas. El grupo "otros" fue por sí solo el más frecuente en esta serie $(41.5 \%)$.

\section{Gráfico 2. Distribución de pacientes confirmados con diagnóstico de influenza por AH1N1 según grupo etáreo y factores de riesgo, Hospital San Juan de Dios, agosto 2009 a julio 2010.}

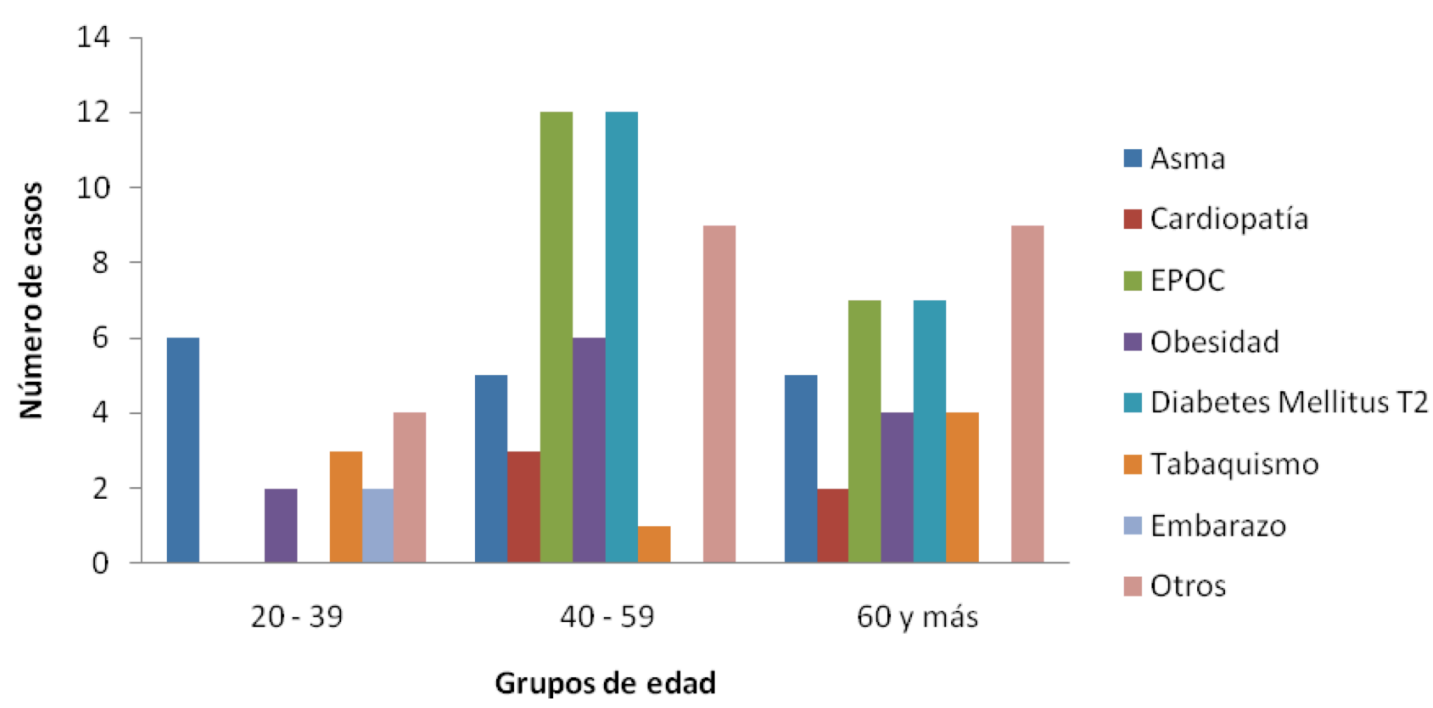

En la distribución por sexo de los factores de riesgo (gráfico 3), se observó que las mujeres padecieron más de asma $(\mathrm{n}=11)$ y diabetes mellitus tipo $2(n=11)$ que el grupo masculino. Por otra parte, el grupo masculino fue el único que presentó fumadores y el que más tuvo pacientes con diagnóstico de EPOC (5 de un total de 7 pacientes), cardiopatías (4 de 5 pacientes) y obesidad $(n=8)$.

\section{DISCUSIÓN}

La distribución de los pacientes internados y confirmados en el HSJD con el diagnóstico de influenza por $\mathrm{AH} 1 \mathrm{~N} 1$ concuerda con la curva epidémica descrita para los casos confirmados por AH1N1 en Costa Rica ${ }^{(4)}$. De acuerdo con la información publicada en el Boletín Epidemiológico 31, se puede observar que la primera "Hola Pandémica" en Costa Rica tuvo su punto final alrededor de las semanas 39 y 42 del año $2009^{(4)}$. El patrón de curva propagada observado en el gráfico 1 es consecuente con el tipo de transmisión de la influenza por $\mathrm{AH} 1 \mathrm{~N} 1^{(5)}$. 


\section{Gráfico 3. Distribución de pacientes confirmados con diagnóstico de influenza por AH1N1 según sexo y factores de riesgo, Hospital San Juan de Dios, agosto 2009 a julio 2010.}

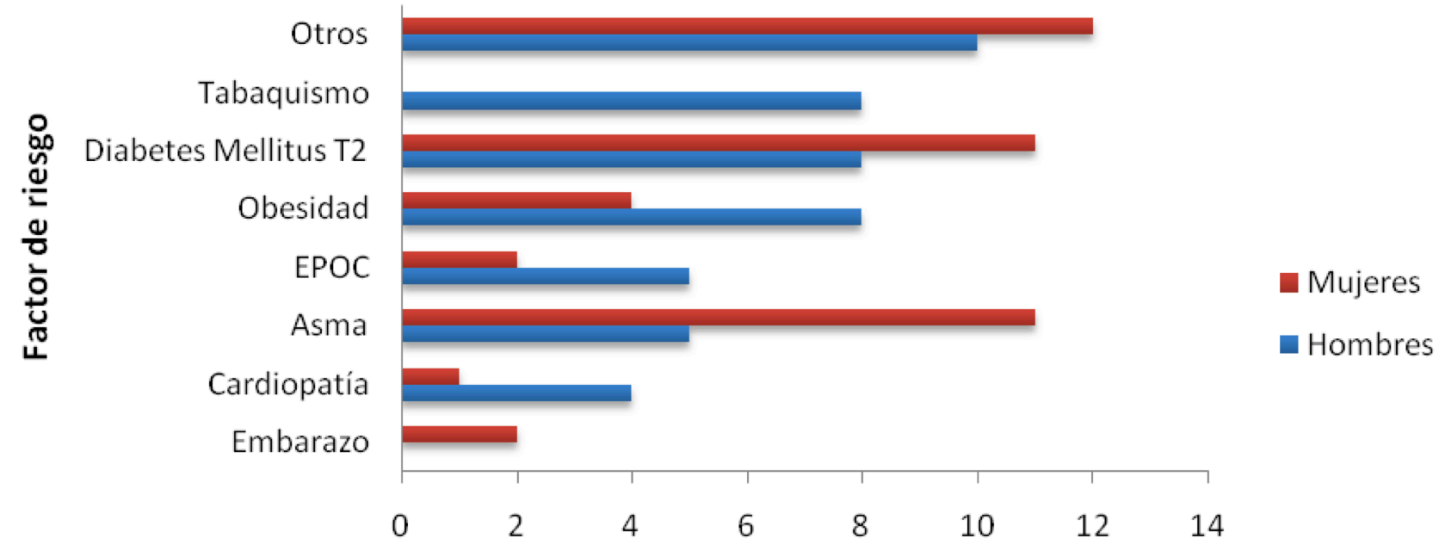

Número de casos

Tabla 1. Casos confirmados de Influenza AH1N1 según grupo de edad y sexo, en el HSJD: agosto 2009 - julio 2010.

\begin{tabular}{|cc|c|c|c|}
$\begin{array}{c}\text { Grupo de } \\
\text { Edad } \\
(\text { años })\end{array}$ & $\begin{array}{c}\text { Frecuencia } \\
\text { absoluta }\end{array}$ & $\begin{array}{c}\text { Frecuencia } \\
\text { relativa }\end{array}$ & $\begin{array}{c}\text { Frecuencia } \\
\text { absoluta }\end{array}$ & $\begin{array}{c}\text { Frecuencia } \\
\text { relativa }\end{array}$ \\
\hline $20-39$ & 11 & $20.75 \%$ & 7 & $13.21 \%$ \\
\hline $40-59$ & 8 & $15.09 \%$ & 11 & $20.75 \%$ \\
\hline 60 y más & 8 & $15.09 \%$ & 8 & $15.09 \%$ \\
\hline Total & 27 & $50.94 \%$ & 26 & $49.06 \%$ \\
\hline
\end{tabular}

Fuente: REMES, HSJD

La razón de masculinidad de toda la población y entre los diferentes grupos de edad fue diferente a lo encontrado a nivel nacional. En el HSJD esta razón fue casi de uno a uno, mientras que en la mayoría de grupos de edad en el país, hubo más hombres que mujeres confirmados con este diagnóstico $^{(4)}$.

Todas las series de pacientes hospitalizados revisadas $^{(6-8)}$ incluyen población pediátrica dentro de sus estudios, a diferencia de esta caracterización, la cual se basa únicamente en población adulta. En el caso de la descripción realizada por Louie y colaboradores $^{(8)}$, se mencionó que la mediana de la edad para el grupo mayor de 18 años en casos no fatales fue de 38 años, con un límite superior de 92 años. La mediana de la edad en casos fatales fue de 46 años con un límite superior de 85 años. En sendos casos, esta cifra fue menor que la encontrada en el HSJD.

La mediana del tiempo que transcurrió entre el inicio de síntomas y el ingreso al HSJD (3 días) y el intervalo intercuartil ( 2 a 7 días) se asemeja a los hallazgos de las series descritas por Santa Olalla $^{(6)}$ y Louie $^{(8)}$ y sendos colaboradores. La primera presentó una mediana de 3 días con un intervalo intercuartil de 2 a 6 días para pacientes internados en la unidad de cuidados intensivos y de 1 a 5 días para los pacientes internados en otros salones. Louie y colaboradores indicaron que los pacientes en California tuvieron una mediana de 2 días, con un rango de 0 a 31 días.

Los factores de riesgo encontrados en los pacientes confirmados con el virus de la Influenza AH1N1 en Costa Rica fueron similares a los factores de riesgo encontrados en otros países $^{(6-9)}$. En el caso particular del HSJD, se evidenció la presencia de patologías pulmonares (EPOC y asma) como las comorbilidades más 
frecuentes dentro de todos los grupos. Entre ambas abarcaron un $43 \%$ de las comorbilidades diagnosticadas. El grupo de vigilancia epidemiológica Europeo describió que en la población de pacientes hospitalizados en España $^{(6)}$, las comorbilidades encontradas más frecuentemente fueron las enfermedades pulmonares crónicas $(35.5 \%)$ y la obesidad mórbida (14.1\%). Este grupo estudió un total de 3025 pacientes. Los pacientes del HSJD tampoco difieren de lo reportado en la literatura internacional $^{(6,8,9)}$ en cuanto al diagnóstico de obesidad $(n=12)$ la cual se observó en un $22.6 \%$ de los casos y se describe como un factor de riesgo frecuentemente encontrado en los pacientes internados y confirmados con influenza por AH1N1.

En la serie de pacientes estudiados en el HSJD, la diabetes mellitus $(n=19)$ fue la enfermedad concomitante al diagnóstico de influenza por AH1N1 diagnosticada con mayor frecuencia. Este hallazgo ha sido descrito como factor de riesgo en algunos grupos estudiados pero no como el más importante ${ }^{(6,8)}$. Esta diferencia podría deberse a que las series revisadas incluyen la descripción de la población pediátrica.

Se puede concluir que con excepción de la diabetes mellitus tipo 2 , la frecuencia relativa de los factores de riesgo encontrados en los pacientes del HSJD se comportaron de forma similar a los hallados en otros países ${ }^{(6-9,11,12)}$.

La mayor limitante para el análisis de este brote fue que la recolección sistemática y estandarizada de los factores de riesgo de los pacientes hospitalizados se llevó a cabo desde el mes de agosto de 2009. Esta situación hizo que por factores de tiempo no se pudieran contemplar para el análisis los pacientes hospitalizados durante los meses de abril, mayo, junio y julio de 2009, meses que correspondieron a la fase de ascenso y pico de la primera hola pandémica en Costa $\operatorname{Rica}^{(4)}$.

Se recomienda ampliar el análisis de los pacientes confirmados a aquellos que fueron internados entre los meses de abril a julio del año 2009 y también estudiar con mayor detalle el grupo de "otros" factores de riesgo ya que fue el encontrado con mayor frecuencia y podría evidencias aspectos importantes sobre el comportamiento de esta enfermedad.

\section{REFERENCIAS BIBLIOGRÁFICAS}

1. World Health Organization (WHO). Swine influenza. 25 de abril 2009. En: http://www.who.int/mediacentre/news/s tatements/2009/h1n1_20090425en/index.ht lm. Accesado: 4 de octubre de 2010.

2. AP. México confirma 260 casos de gripe AH1N1: se han procesado 547 pruebas de influenza. El Espectador.com. 30 de abril de 2009. http://www.elespectador.com/noticias/s alud/articulo138701-mexico-confirma260-casos-de-gripe-ah1n1 Accesado: 4 de octubre de 2010.

3. Rodríguez, I. Gripe AH1N1 cumple un año con más preguntas que respuestas. La Nación. Suplemento Aldea Global. 22 de abril de 2009. http://www.nacion.com/2010-0423/AldeaGlobal/NotaPrincipal/AldeaG1 obal2344075.aspx Accesado: 4 de octubre de 2010.

4. Ministerio de Salud Costa Rica. Boletín Semanal de Vigilancia de la Salud: semana epidemiológica 31.

5. Torok M. Enfoque en epidemiología de campo: curvas epidémicas. Escuela de Salud Pública, Universidad de Carolina del Norte. 1(5):2-6.

6. Santa-Olalla P Cortés-García M Vicente-Herrero $\mathrm{M}$ et al. Risk factors for disease severity among hospitalized patients with 2009 pandemic influenza A(H1N1) in Spain, April-December 2009. Eurosurveillance. 2010;15(38).

7. Rey-Benito J Castro-Jiménez M Casti1lo-Pavón J. Análisis descriptivo de las primeras muertes por influenza pandémica (H1N1) 2009 en Colombia. Asociación Colombiana de Infectología. 2010;13(4):254-258.

8. Louie J Acosta M Winter K et al. Factors associated with death or hospitalization due to pandemic 2009 influenza A (H1N1) infection in California. JAMA. 2009;302(17):1896-1902.

9. Chowell G Bertozzi S Colchero A et al. Severe respiratory disease concurrent with the circulation of H1N1 influenza. N Eng J Med. 2009;361:674-679.

10. Girard M Tam J Assossou O Kieny M. The 2009 A (H1Nl) influenza virus 
pandemic: a review. Vaccine. 2010;28 (31):4895-4902.

11. Yates L Pierce M Stephens S et al. Influenza A/H1N1v in pregnancy: an investigation of the characteristics and management of affected women and the relationship to pregnancy outcomes for mother and infant. Health Technol Assess. 2010; 14(34):109-182.

12. Toal M Agyeman-Duah K Schwenk A

Yoong WJ. Swine flu and pregnancy. J

Obstet Gynaecol. 2010;30(2):97-100. 